\title{
Energy-efficient uplink power control for multiuser SIMO systems with imperfect channel state information
}

\author{
Moonheok Jang, Younggap Kwon, Hyunsung Park and Taewon Hwang ${ }^{*}$
}

\begin{abstract}
This paper addresses energy-efficient design for uplink multiuser SIMO systems with imperfect channel state information (CSI) at the base station (BS). Since the CSI at the BS is always imperfect due to the channel estimation error and delay, the imperfectness of the CSI needs to be considered in practical system design. It causes interuser interference at the zero-forcing (ZF) receiver and makes it difficult to obtain the globally optimal power allocation that maximizes the energy efficiency (EE). Hence, we propose a non-cooperative energy-efficient uplink power control game, where each user selfishly updates its own uplink power. The proposed uplink power control game is shown to admit a unique Nash equilibrium. Furthermore, to improve the efficiency of the Nash equilibrium, we study a new game that utilizes a pricing mechanism. For the new game, the existence of a Nash equilibrium and the convergence of the best response dynamics are studied based on super-modularity theory. Simulation results show that the proposed schemes can significantly improve the EEs of the mobile users in uplink multiuser SIMO systems.
\end{abstract}

Keywords: Energy efficiency; Multiuser; Multiple-input and multiple-output; Uplink; Channel state information; Game theory; Nash equilibrium

\section{Introduction}

Multiple-input multiple-output (MIMO) has been considered as one of the key technologies for wireless communication systems due to its potential to achieve high spectral efficiency (SE) as well as increased diversity and interference suppression [1]. For this reason, many research on MIMO has focused on increasing the SE [2-6]. On the other hand, the rapid increase in the wireless data traffic has caused dramatic increase in energy consumption of wireless communications, which results in massive greenhouse gas emission and high operation cost [7]. Thus, energy-efficient communication system design is becoming more important in preserving the environment and reducing operation cost. Moreover, energy-efficient design is also important for prolonging the battery life because the development of battery technology has not kept up with the increasing demand on the energy supply for the mobile communications.

*Correspondence: twhwang@yonsei.ac.k

School of Electrical and Electronic Engineering, Yonsei University, 120-749 Seoul, Korea
For this reason, recent research on MIMO systems has also considered energy-efficient designs [8-19] as well as spectral-efficient designs. For example, in [8], a single-user MIMO system, where the MIMO channel is converted to parallel independent subchannels through singular value decomposition (SVD) and then the transmit power is allocated across the subchannels to maximize the energy efficiency (EE) of the system, is considered. In [9], an energy-efficient precoder design is investigated according to the type of fading, i.e., static, fast, and slow fading. In [10], power allocation and antenna selection are jointly optimized to maximize the EE. In [11], the uplink of a MIMO system is considered, and a mechanism for the mobile terminals to switch between MIMO and singleinput multiple-output (SIMO) to increase their EE is proposed. In [12], the downlink of a multiuser MIMO system is considered, and the optimal power allocation that maximizes the EE of the base station (BS), which employs zero-forcing (ZF) beamforming, is designed. In [13], the EE capacity for uplink multiuser MIMO system is defined, and a low-complexity uplink power allocation algorithm that achieves this capacity is proposed. In [15], the optimal

\section{照 Springer}

(c) 2014 Jang et al: licensee Springer. This is an Open Access article distributed under the terms of the Creative Commons

Attribution License (http://creativecommons.org/licenses/by/2.0), which permits unrestricted use, distribution, and reproduction in any medium, provided the original work is properly credited. 
number of mobile users in uplink multiuser MIMO systems and the optimal power allocation that maximize the $\mathrm{EE}$ are discussed. In [17], the energy-efficient link adaptation for uplink coordinated multi-point (CoMP) systems is investigated.

The above research assumes perfect channel state information (CSI) at the transmitters and/or the receivers. However, CSI is always imperfect due to channel estimation error and delay, and therefore, it is important to consider the impact of imperfect CSI for practical wireless communication system design. So far, only a few research has considered imperfect CSI in EE design for MIMO. In [20], energy-efficient subcarrier and power allocation in the uplink of a multi-carrier interference network are addressed, where only statistical CSI is available at the transmitters. In [21], bandwidth, transmit power, active transmit/receive antenna number, and active user number are adjusted to improve the system-wise energy efficiency in the downlink multiuser MIMO systems assuming imperfect CSI at the BS.

In this paper, we address energy-efficient power control of uplink multiuser SIMO systems with imperfect CSI at the BS. The imperfect CSI causes interuser interference at the ZF receiver and makes it difficult to obtain the globally optimal power allocation that maximizes the EE. Hence, instead of using a conventional optimization-theoretic approach, we propose a non-cooperative energy-efficient uplink power control game, where each user selfishly updates its own uplink power to maximize its own EE. It is shown that the proposed uplink power control game admits a unique Nash equilibrium. Furthermore, to improve the efficiency of the Nash equilibrium, we study a new game that utilizes a pricing mechanism. For the new game, the existence of a Nash equilibrium and the convergence of the best response dynamics are studied based on super-modularity theory. Simulation results show that the proposed schemes can significantly improve the EEs of the mobile users in uplink multiuser SIMO systems.

The rest of the paper is organized as follows: In Section 2, we describe the system model. In Section 3, we define the EE of each mobile user. In Section 4, a noncooperative energy-efficient uplink power control game is formulated, the existence and the uniqueness of the Nash equilibrium are discussed, and a pricing mechanism is introduced to improve the efficiency of the Nash equilibrium. The numerical results are reported in Section 5, while the concluding remarks are given in Section 6.

Notations: Superscripts $(\cdot)^{T},(\cdot)^{*}$, and $(\cdot)^{H}$ stand for transpose, complex conjugate, and complex conjugate transpose operations, respectively. Uppercase boldface letters are used to denote matrices, whereas lowercase boldface letters are used to denote vectors. I stands for an identity matrix. $\|\mathbf{x}\|=\sqrt{\mathbf{x}^{H} \mathbf{x}} ; \mathcal{C N}$ $\left(\mathbf{0}, \sigma^{2} \mathbf{I}\right)$ denotes zero-mean circularly symmetric, complex
Gaussian distribution with covariance matrix $\sigma^{2} \mathbf{I}$. $E[\cdot]$ represents expectation. $[\mathbf{A}]_{i j}$ signifies the $i$-th row, $j$-th column element of matrix $\mathbf{A} . \mathbf{x} \succeq \mathbf{y}$ denotes componentwise inequality between vectors $\mathbf{x}$ and $\mathbf{y} \cdot \operatorname{proj}_{\mathcal{V}} \mathbf{x}$ denotes the projection of a vector $\mathbf{x}$ on a subspace $\mathcal{V}$.

\section{System model}

Consider the uplink of a multiuser SIMO system consisting of a BS and $K$ users, where the BS has $M$ antennas while each user has a single antenna. The received signal vector at the $\mathrm{BS}$ can be expressed as

$$
\mathbf{y}=\sum_{k=1}^{K} \sqrt{\beta_{k}} \mathbf{h}_{k} x_{k}+\mathbf{n}
$$

where $\sqrt{\beta_{k}}$ is the large-scale fading coefficient from the $k$ th user to the BS assumed to be known a priori, $\mathbf{h}_{k} \sim$ $\mathcal{C N}(\mathbf{0}, \mathbf{I})$ is the $M \times 1$ channel vector from the $k$ th user to the BS, $x_{k}$ is the symbol of the $k$ th user, and $\mathbf{n} \sim \mathcal{C N}\left(\mathbf{0}, \sigma^{2} \mathbf{I}\right)$ is the $M \times 1$ zero-mean additive white Gaussian noise (AWGN) vector.

The BS estimates $\mathbf{h}_{k}$ 's using the minimum mean square error (MMSE) estimator. Also, there exists a delay between channel estimation and its actual use. From [22], the relationship between the actual channel $\mathbf{h}_{k}$ and its estimate $\hat{\mathbf{h}}_{k}$ can be written as

$$
\mathbf{h}_{k}=\rho_{k} \hat{\mathbf{h}}_{k}+\mathbf{e}_{k}
$$

where $\rho_{k}$ is the correlation coefficient between the actual channel and its estimate and $\mathbf{e}_{k} \sim \mathcal{C N}\left(\mathbf{0},\left(1-\rho_{k}^{2}\right) \mathbf{I}\right)$. Note that $\hat{\mathbf{h}}_{k}$ and $\mathbf{e}_{k}$ are Gaussian and orthogonal to each other from the orthogonality principle [23], which implies that $\hat{\mathbf{h}}_{k}$ and $\mathbf{e}_{k}$ are independent. Then, the BS applies zero-forcing receiver $\mathbf{A}=\left(\hat{\mathbf{H}}^{H} \hat{\mathbf{H}}\right)^{-1} \hat{\mathbf{H}}^{H}$ based on the estimated channel $\hat{\mathbf{H}}=\left[\hat{\mathbf{h}}_{1}, \ldots, \hat{\mathbf{h}}_{K}\right]$. Denote $\mathbf{a}_{k}^{T}$ to be the normalized version of the $k$ th row of $\mathbf{A}$. Then, the ZF receiver output for the $k$ th user can be written as

$$
\begin{aligned}
r_{k}= & \overbrace{\sqrt{\beta_{k}} \rho_{k} \mathbf{a}_{k}^{T} \hat{\mathbf{h}}_{k} x_{k}}^{\text {signal }}+\overbrace{\sqrt{\beta_{k}} \mathbf{a}_{k}^{T} \mathbf{e}_{k} x_{k}}^{\text {intersymbol interference }} \\
& +\underbrace{\sum_{j=1, j \neq k}^{K} \sqrt{\beta_{j}} \mathbf{a}_{k}^{T} \mathbf{e}_{j} x_{j}}_{\text {interuser interference }}+\underbrace{n_{k}^{\prime}}_{\text {noise }},
\end{aligned}
$$

where $n_{k}^{\prime}=\mathbf{a}_{k}^{T} \mathbf{n}$. Note that there exist both interuser interference and intersymbol interference due to the channel estimation error. The instantaneous signal-to- 
interference-plus-noise ratio (SINR) of the $k$ th user can be written as

$$
\gamma_{k}=\frac{c_{k} \rho_{k}^{2}\left|\mathbf{a}_{k}^{T} \hat{\mathbf{h}}_{k}\right|^{2} p_{k}}{c_{k}\left|\mathbf{a}_{k}^{T} \mathbf{e}_{k}\right|^{2} p_{k}+\sum_{j=1, j \neq k}^{K} c_{j}\left|\mathbf{a}_{k}^{T} \mathbf{e}_{j}\right|^{2} p_{j}+1},
$$

where $p_{k}=E\left[\left|x_{k}\right|^{2}\right]$ and $c_{k}=\beta_{k} / \sigma^{2}$ are transmission power and the channel-to-noise ratio (CIR) of the $k$ th user, respectively. Then, the instantaneous rate of the $k$ th user is given by ${ }^{\mathrm{a}} r_{k}=\log \left(1+\gamma_{k}\right)$.

\section{Energy efficiency}

To obtain the distribution of $\gamma_{k}$, we consider the following properties, which are proved in [24].

Property 1 . Consider a $M \times 1$ Gaussian random vector $\mathbf{g} \sim \mathcal{C N}(\mathbf{0}, \mathbf{I})$ and a $M \times 1$ unit norm random vector $\mathbf{u}(\|\mathbf{u}\|=1)$ which is independent of $\mathbf{g}$. Then, $\left|\mathbf{g}^{T} \mathbf{u}\right|^{2} \sim$ $\operatorname{Exp}(1)$ where $\operatorname{Exp}(\theta)$ denotes exponential distribution with mean $\theta$.

Property 2. Consider a vector space $\mathcal{V}$ with $\operatorname{dim}(\mathcal{V})=$ $m$. Also, define $\mathbf{a}=\frac{\mathbf{b}}{\|\mathbf{b}\|}$, where $\mathbf{b}=\operatorname{proj}_{\mathcal{V}} \mathbf{h}$, and assume the elements of $\mathbf{h}$ are i.i.d complex Gaussian random variables with unit variance. Then, $\left|\mathbf{h}^{H} \mathbf{a}\right|^{2} \sim \frac{1}{2} \chi_{2 m}^{2}$ where $\chi_{\theta}^{2}$ denotes chi-square distribution with degree of freedom $\theta$.

Using the above properties, we have

$$
\left|\mathbf{a}_{k}^{T} \mathbf{e}_{j}\right|^{2} \sim\left(1-\rho_{j}^{2}\right) \operatorname{Exp}(1), j=1, \ldots, K,
$$

and

$$
\left|\mathbf{a}_{k}^{T} \hat{\mathbf{h}}_{k}\right|^{2} \sim \frac{1}{2} \chi_{2(M-K+1)}^{2}, \quad k=1, \ldots, K .
$$

Assuming that the channel is ergodic so that each codeword spans over a large number of realization of the small-scale fading channel, the ergodic achievable rate of the $k$ th user $R_{k}$ is given by

$$
R_{k}=E\left[r_{k}\right]=E\left[\log \left(1+\gamma_{k}\right)\right]
$$

Since finding a closed form expression of (5) is not easy, we resort to a lower bound of $R_{k}$ obtained in the following theorem.

Theorem 1. A lower bound of the ergodic rate of the $k$ th user in (5) is given by

$$
R_{k} \geq R_{k}^{\text {lower }}=\log \left(1+\frac{s_{k} p_{k}}{i_{k} p_{k}+\sum_{j=1, j \neq k}^{K} i_{j} p_{j}+1}\right),
$$

where $s_{k}=(M-K) c_{k}\left|\rho_{k}\right|^{2}$ and $i_{k}=c_{k}\left(1-\left|\rho_{k}\right|^{2}\right)$, for $k=1, \ldots, K$.

\section{Proof. See Appendix 1.}

Note that the above lower bound assumes the regime where the number of BS antennas exceeds the number of user, i.e., $M>K$. Many recent researches advocate using sufficiently large number of antennas at the BS to increase EE as well as SE [25]. Using large number of antennas at the $\mathrm{BS}$ can reduce the transmit power of the mobile users in the uplink and slow down the battery power consumption. For example, in massive MIMO, the BS employs massive number of antennas, say a hundred or a few hundreds of antennas, to improve the EE of users or BS [26].

The EE of the $k$ th user is defined as the average number of information bits that can be reliably conveyed over the channel per unit energy consumption, i.e.,

$$
\eta_{k}=\frac{R_{k}}{p_{k}+p_{c}}
$$

where $p_{c}$ denotes the circuit power which is independent of the transmission power [27]. Using the lower bound of $R_{k}$, the EE $\eta_{k}$ can be approximated by

$$
\eta_{k} \approx \frac{\log \left(1+\frac{s_{k} p_{k}}{i_{k} p_{k}+I_{k}}\right)}{p_{k}+p_{c}}
$$

where $I_{k}=\sum_{j=1, j \neq k}^{K} i_{j} p_{j}+1$.

The EE of the uplink multiuser SIMO system $\eta$ is defined as the sum of the EEs of the users, i.e., $\eta=\sum_{k=1}^{K} \eta_{k}$, which is a function of $\left\{p_{k}\right\}_{k=1}^{K}$. To maximize the system $\mathrm{EE}$, we need to solve the following problem:

$$
\begin{aligned}
& \underset{\mathbf{p}}{\operatorname{maximize}} \eta \\
& \text { subject to } 0 \leq p_{k} \leq p_{\max }, \quad \forall k=1, \ldots, K
\end{aligned}
$$

where $\mathbf{p}=\left[p_{1}, \ldots, p_{K}\right]^{T}$ denotes the uplink power vector of the users. Finding the optimal $\mathbf{p}$ that maximizes the system EE using the conventional optimization theory is difficult because the objective function $\eta$ is not concave in p. Larger number of users $K$ in the system will result in more local maximums, and searching for the globally optimal power allocation for the users would be a daunting task. Hence, in this paper, we consider a game theoretic approach, where each user finds its own uplink power in a distributed fashion ${ }^{\mathrm{b}}$. 


\section{Energy-efficient uplink power control based on pricing}

Denote $\mathbf{p}_{-k}=\left[p_{1}, \ldots, p_{k-1}, p_{k+1}, \ldots, p_{K}\right]^{T}$ to be the power vector of the other users than the $k$ th user. Then, the non-cooperative energy-efficient uplink power control game $\mathcal{G}$ can be written as

$$
\mathcal{G}\left(\mathcal{K},\left\{\mathcal{Q}_{k}\right\}_{k=1}^{K},\left\{\eta_{k}\left(p_{k}, \mathbf{p}_{-k}\right)\right\}_{k=1}^{K}\right),
$$

where $\mathcal{K}=\{1, \ldots, K\}$ is the set of players (users), $\mathcal{Q}_{k}=$ $\left\{p_{k} \mid 0 \leq p_{k} \leq p_{\max }\right\}$ is the set of strategies (power levels) available to the $k$ th user, and $\eta_{k}\left(p_{k}, \mathbf{p}_{d,-k}\right)$ is a utility function (EE) the $k$ th user seeks to maximize. The game $\mathcal{G}$ can be also expressed as the following $K$ coupled problems.

$$
\begin{aligned}
& \underset{p_{k}}{\operatorname{maximize}} \eta_{k}\left(p_{k}, \mathbf{p}_{-k}\right) k=1, \ldots, K \\
& \text { subject to } p_{k} \in \mathcal{Q}_{k}, \quad k=1, \ldots, K .
\end{aligned}
$$

Given the uplink power of the other users, $\mathbf{p}_{-k}$, the best response of the $k$ th user is given by

$$
p_{k}^{o}=B_{k}\left(\mathbf{p}_{-k}\right) \triangleq \arg \max _{0 \leq p_{k} \leq p_{\max }} \eta_{k}\left(p_{k}, \mathbf{p}_{-k}\right),
$$

where $\eta_{k}$ is a function of both $p_{k}$ and $\mathbf{p}_{-k}$. The variation of uplink power of one user impacts those of the other users. Equilibrium is the condition in which competing influences are balanced. The most commonly used solution concept in game theory is that of the Nash equilibrium [28]. A Nash equilibrium for the game $\mathcal{G}$ can be described as a fixed point of the following non-linear equation:

$$
\mathbf{p}^{*}=B\left(\mathbf{p}^{*}\right)
$$

where $B\left(\mathbf{p}^{*}\right) \triangleq\left[B_{1}\left(\mathbf{p}_{-1}^{*}\right), B_{2}\left(\mathbf{p}_{-2}^{*}\right), \ldots, B_{K}\left(\mathbf{p}_{-K}^{*}\right)\right]^{T}$. For more details on the Nash equilibrium, we refer interesting readers to [28].

The game $\mathcal{G}$ has a unique Nash equilibrium. The existence and the uniqueness of the Nash equilibrium can be shown by using the results in [29] because the utility in (8) has the same form as the one used in [29]. Note that [29] discusses a game in a relay-assisted network where $K$ transmitter-receiver pairs communicate by means of an AF relay. If the direct links between the transmitters and the receivers are ignored, the SINR expression in [29] has the same form as the SINR in (2). An interesting characteristic in the SINR expressions in (2) and [29] is that both SINR expressions have a common term in the denominator that depends on the $k$ th user's signal power (or the $k$ th transmitter's signal power) $p_{k}$. While this term is due to the channel estimation error in (2), it is due to the transmit power normalization at the relay in [29].

Now, we discuss the techniques on how to improve the efficiency of the Nash equlibrium of the game $\mathcal{G}$. In the game $\mathcal{G}$, each player only aims to maximize its own EE by adjusting its own power, but it ignores the interference it generates to the other players. Thus, the Nash equilibrium of the game $\mathcal{G}$ may be inefficient in the Pareto sense [30]. We say that a strategy profile $\mathbf{p}_{1}$ is more efficient than another strategy profile $\mathbf{p}_{2}$ if, for all $k \in \mathcal{K}, \eta_{k}\left(\mathbf{p}_{1}\right) \geq \eta_{k}\left(\mathbf{p}_{2}\right)$ and for some $k \in \mathcal{K}, \eta_{k}\left(\mathbf{p}_{1}\right)>$ $\eta_{k}\left(\mathbf{p}_{2}\right)$.

To improve the efficiency of the Nash equilibrium of the game $\mathcal{G}$, we study a new game with the pricing mechanism, namely game $\mathcal{G}^{c}$. By introducing pricing to the player's utility functions, the player now voluntarily cooperates with each other to improve the system performance. We adopt a simple linear pricing policy that each player needs to pay the price that linearly increases with the amount of uplink power consumption. The new game $\mathcal{G}^{c}$ can be expressed as

$$
\mathcal{G}^{c}\left(\mathcal{K},\left\{\mathcal{Q}_{k}\right\}_{k=1}^{K},\left\{\eta_{k}^{c}\left(p_{k}, \mathbf{p}_{-k}\right)\right\}_{k=1}^{K}\right),
$$

where

$$
\eta_{k}^{c}\left(p_{k}, \mathbf{p}_{-k}\right)=\eta_{k}\left(p_{k}, \mathbf{p}_{-k}\right)-c p_{k}
$$

is the utility of the $k$ th player that incorporates pricing factor $c>0$. Then, we discuss the existence of a Nash equilibrium of the game $\mathcal{G}^{c}$. Note that $\eta_{k}^{c}$ is the sum of two quasi-concave functions, which is not necessarily quasi-concave. Here, we resort to super-modularity theory [31] to show the existence of a Nash equilibrium. The formal definition of a supermodular game is provided below.

Definition 1. A non-cooperative game $\mathcal{G}^{c}\left(\mathcal{K},\left\{\mathcal{Q}_{k}\right\}_{k=1}^{K}\right.$, $\left.\left\{\eta_{k}^{c}\left(p_{k}, \boldsymbol{p}_{-k}\right)\right\}_{k=1}^{K}\right)$ is a supermodular game if for all $k \in \mathcal{K}$,

1. $\mathcal{Q}_{k}$ is a compact subset of $\mathbf{R}$

2. $\eta_{k}^{c}\left(p_{k}, \boldsymbol{p}_{-k}\right)$ is upper semi-continuous in $\boldsymbol{p}$

3. For all $\boldsymbol{p}_{-k} \succeq \boldsymbol{p}_{-k}^{\prime}$, the quantity $\eta_{k}^{c}\left(p_{k}, \boldsymbol{p}_{-k}\right)-\eta_{k}^{c}\left(p_{k}, \boldsymbol{p}_{-k}^{\prime}\right)$ is non-decreasing in $p_{k} \in \mathcal{Q}_{k}$.

The game $\mathcal{G}^{c}$ in (13) satisfies conditions 1) and 2), but it violates condition 3). Therefore, the game $\mathcal{G}^{c}$ is not a supermodular game. However, if the strategy spaces of players are modified appropriately according to Theorem 2, the resulting game becomes supermodular.

Theorem 2. Denote the modified strategy space for the kth user as $\mathcal{Q}_{k}^{c} \triangleq\left\{p_{k} \mid p_{\min , k} \leq p_{k} \leq p_{\max }\right\}$, where

$$
p_{\min , k}=\frac{i_{k}+\sqrt{i_{k}^{2}+2 s_{k} i_{k} I_{\max , k}}}{s_{k} i_{k}}
$$


with $I_{\max , k}=\sum_{i=1, i \neq k}^{K} i_{j} p_{\max }+1$. Then, the modified energyefficient uplink power control game with pricing

$$
\mathcal{G}^{c}\left(\mathcal{K},\left\{\mathcal{Q}_{k}^{c}\right\}_{k=1}^{K},\left\{\eta_{k}^{c}\left(p_{k}, \boldsymbol{p}_{-k}\right)\right\}_{k=1}^{K}\right)
$$

is a supermodular game.

Proof. See Appendix 2.

It is well known that a supermodular game has at least one Nash equilibrium [31]. Denote $\mathcal{S}_{\mathrm{NE}}$ to be the set of Nash equilibria of a supermodular game. Then, all Nash equilibria of the supermodular game $\mathbf{p}^{*} \in \mathcal{S}_{\mathrm{NE}}$ satisfy $\mathbf{p}_{S}^{*} \preceq \mathbf{p}^{*} \preceq \mathbf{p}_{L}^{*}$, where $\mathbf{p}_{S}^{*}$ and $\mathbf{p}_{L}^{*}$ are the smallest and the largest Nash equilibria in $\mathcal{S}_{\mathrm{NE}}$, respectively. Using the best response dynamics with the smallest strategy vector $\mathbf{p}=\left[\begin{array}{lll}p_{\min , 1} & \ldots & p_{\min , K}\end{array}\right]^{T}$, the strategy vector converges to $\mathbf{p}_{S}^{*}[32]$.

\section{Algorithm 1}

1. Set step size $\Delta c>0$, and initialize pricing factor $c=0$.

2. Set the initial uplink power vector $p_{k}=p_{\min , k}$ for all $k \in \mathcal{K}$.

3. Get $\eta_{k}$ for all $k \in \mathcal{K}$ at the Nash equilibrium of the game $\mathcal{G}^{c}$;

Set $\eta_{k}^{\text {best }}=\eta_{k}$ for all $k \in \mathcal{K}$;

Set $c_{\text {best }}=c$;

4. Increase $c=c_{\text {best }}+\Delta c$.

5. Get $\eta_{k}$ for all $k \in \mathcal{K}$ at the Nash equilibrium of the game $\mathcal{G}^{c}$.

6. If $\eta_{k} \geq \eta_{k}^{\text {best }}$ for all $k \in \mathcal{K}$,

1) Update $\eta_{k}^{\text {best }}=\eta_{k}$ for all $k \in \mathcal{K}$; Update $c_{\text {best }}=c$;

2) Go to Step 4.

\section{Else}

1) Return $c_{\text {best }}$.

2) Terminate algorithm.

Now, we propose an algorithm that finds the pricing factor $c$ that improves the system performance of the game $\mathcal{G}^{c}$ in the Pareto sense, which is summarized in Algorithm 1. The same mechanism in [30] has been used in Algorithm 1 ; we first obtain the utilities $\left\{\eta_{k}\right\}_{k=1}^{K}$ at the Nash equilibrium of the game $\mathcal{G}^{c}$ with no pricing, i.e., $c=0$, which is equivalent to playing the game $\mathcal{G}$. Then, the game $\mathcal{G}^{c}$ is played again after incrementing the price by a positive vale $\Delta c$. If the utilities at this new equilibrium with some positive price $c$ improve with respect to the previous instance, the pricing factor is incremented and the procedure is repeated. This process is repeated until an increase in $c$ results in utility worse than the previous equilibrium values for at least one player. We declare the last value of $c$ with Pareto improvement to be the best pricing factor, $c_{\text {best }}$. As will be shown in our simulation, this technique performs very well in improving the efficiency of the Nash equilibrium.

\section{Numerical results}

In this section, we present the performance of our energyefficient uplink power control scheme obtained by simulations. The system parameters used for simulation are as follows: system bandwidth $W=10 \mathrm{kHz}$, noise power spectral density $N_{0}=-174 \mathrm{dBm} / \mathrm{Hz}$, noise power $\sigma^{2}=$ $N_{0} W=-134 \mathrm{dBm}$, and maximum transmit power of users $p_{\max }=23 \mathrm{dBm}$ and circuit power of users $p_{c}=$ $115.9 \mathrm{~mW}$.

Figure 1 illustrates the convergence of the best response dynamics $\mathbf{p}^{(i+1)}=B\left(\mathbf{p}^{(i)}\right)$ of the game $\mathcal{G}$ when the BS has $M=8$ antennas and there are $K=4$ users. The initial uplink power vector is chosen as $\mathbf{p}^{(0)}=\mathbf{0}$. As shown in the figure, the uplink powers of the users obtained by the best response dynamics converges to the Nash equilibrium within a few iterations. Here, we choose $\rho=0.9995$ when the CSI is imperfect. According to [22], the correlation coefficient between the actual channel and its estimate can be written as $\rho=\rho_{e} \rho_{d}$, where $0 \leq \rho_{e} \leq 1$ and $0 \leq \rho_{d} \leq 1$ are due to the estimation error and the delay, respectively. From Jakes' model, $\rho_{d}=J_{0}\left(2 \pi T_{d} f_{d}\right)$, where $T_{d}$ and $f_{d}$ are the time delay and the maximal Doppler frequency, respectively. Assuming $\rho_{e}=1, T_{d}=1 / 14 \mathrm{~ms}$, and the speed of the user is $5 \mathrm{~km} / \mathrm{h}$, we can obtain 0.9995 .

Next, we compare the energy-efficient uplink power control game $\mathcal{G}$ and the spectral-efficient uplink power control game $\mathcal{G}^{s}$, which can be expressed as

$$
\mathcal{G}^{s}\left(\mathcal{K},\left\{\mathcal{Q}_{k}\right\}_{k=1}^{K},\left\{u_{k}^{s}\left(p_{k}, \mathbf{p}_{-k}\right)\right\}_{k=1}^{K}\right),
$$

where the utility of each player $u_{k}^{s}=R_{k}^{\text {lower }}$ is the lower bound of the ergodic rate in (6). It is clear that the Nash equilibrium of the game $\mathcal{G}^{s}$ is $\mathbf{p}^{*}=\left[p_{\max } \cdots p_{\max }\right]^{T}$ because $R_{k}^{\text {lower }}$ is monotone increasing in $p_{k}$.

Figures 2 and 3 show the cumulative distribution function (CDF) of instantaneous EE $r_{k} /\left(p_{k}+p_{c}\right)$ and the instantaneous rate $r_{k}$ of the $K=4$ users, respectively. As expected, the EE of the proposed scheme is better than that of the spectral-efficient uplink power control scheme for each user as shown in Figure 2. It is interesting that the average rate of the proposed scheme is also better than that of the spectral-efficient uplink power control scheme as shown in Figure 3. This can be explained as 


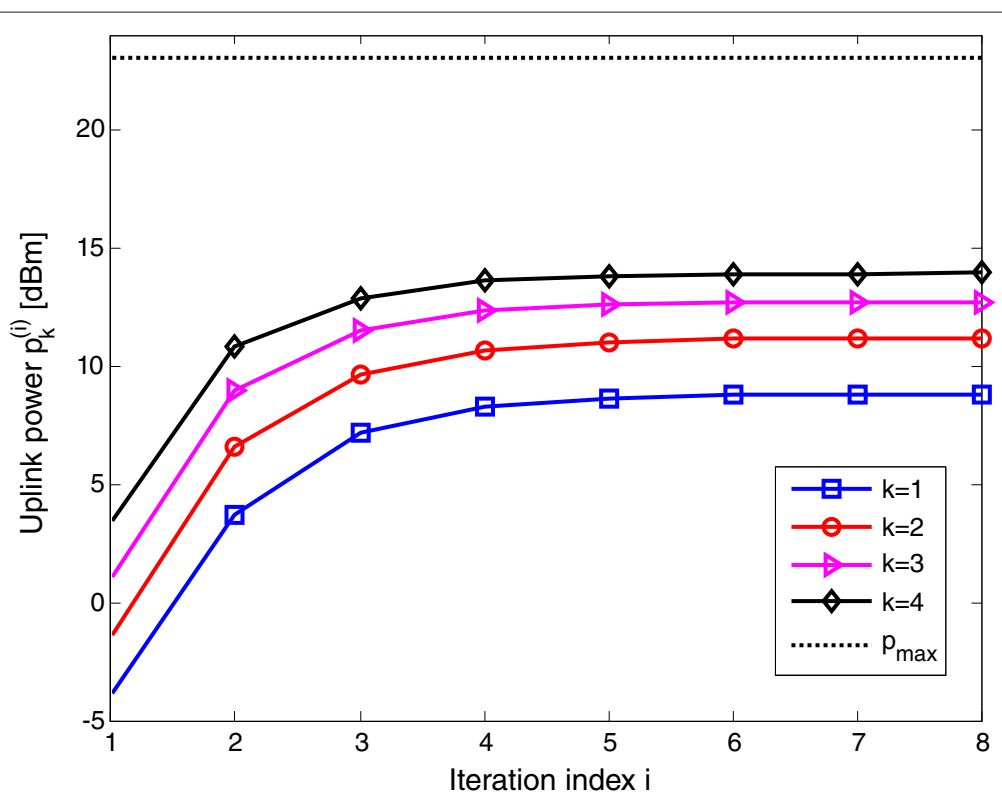

Figure 1 Convergence of the best response dynamics of the game $\mathcal{G} . M=8, K=4$, and $\beta_{1}=-85 \mathrm{~dB}, \beta_{2}=-90 \mathrm{~dB}, \beta_{3}=-95 \mathrm{~dB}$, $\beta_{4}=-100 \mathrm{~dB}$ and $\rho_{1}=\rho_{2}=\rho_{3}=\rho_{4}=0.9995$.

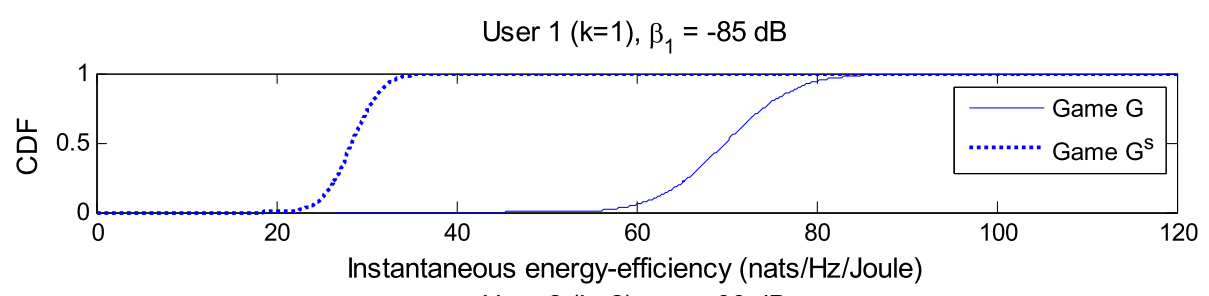

User $2(k=2), \beta_{2}=-90 \mathrm{~dB}$
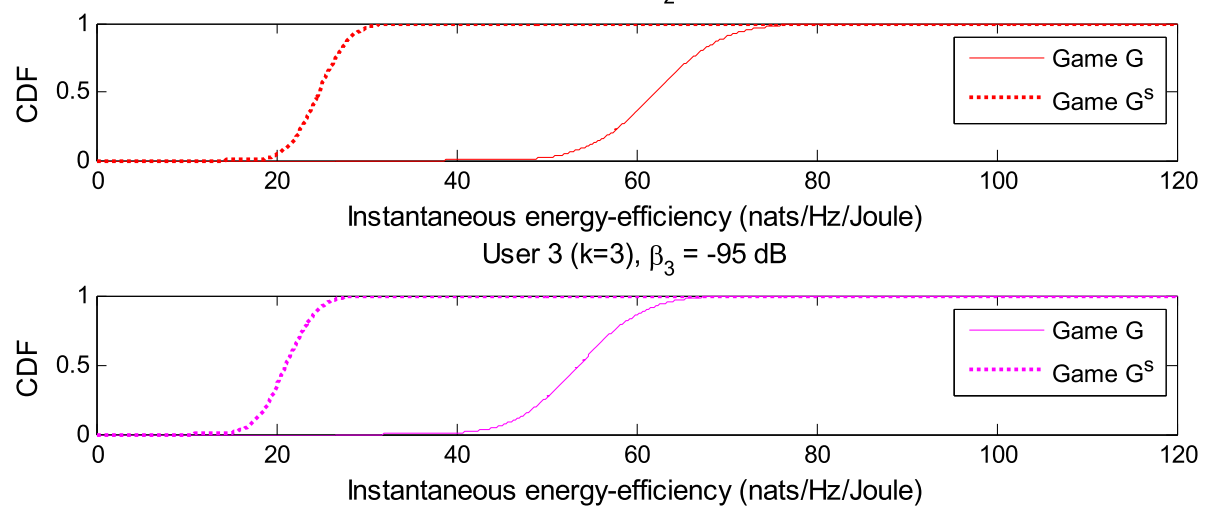

User $4(k=4), \beta_{4}=-100 \mathrm{~dB}$

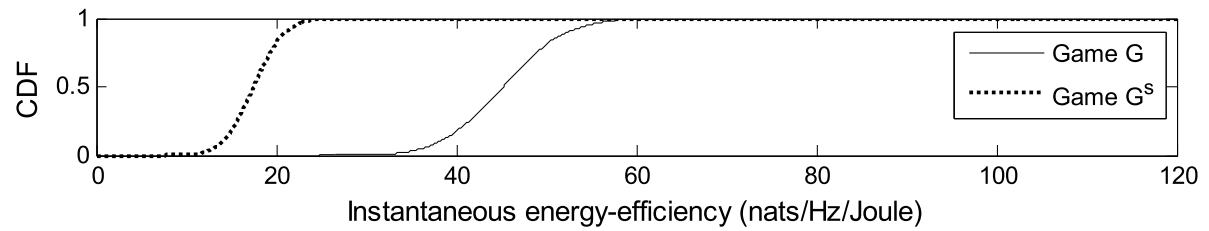

Figure $2 \mathrm{CDF}$ of instantaneous EE. $M=8, K=4$, and $\rho_{1}=\rho_{2}=\rho_{3}=\rho_{4}=0.9995$. 

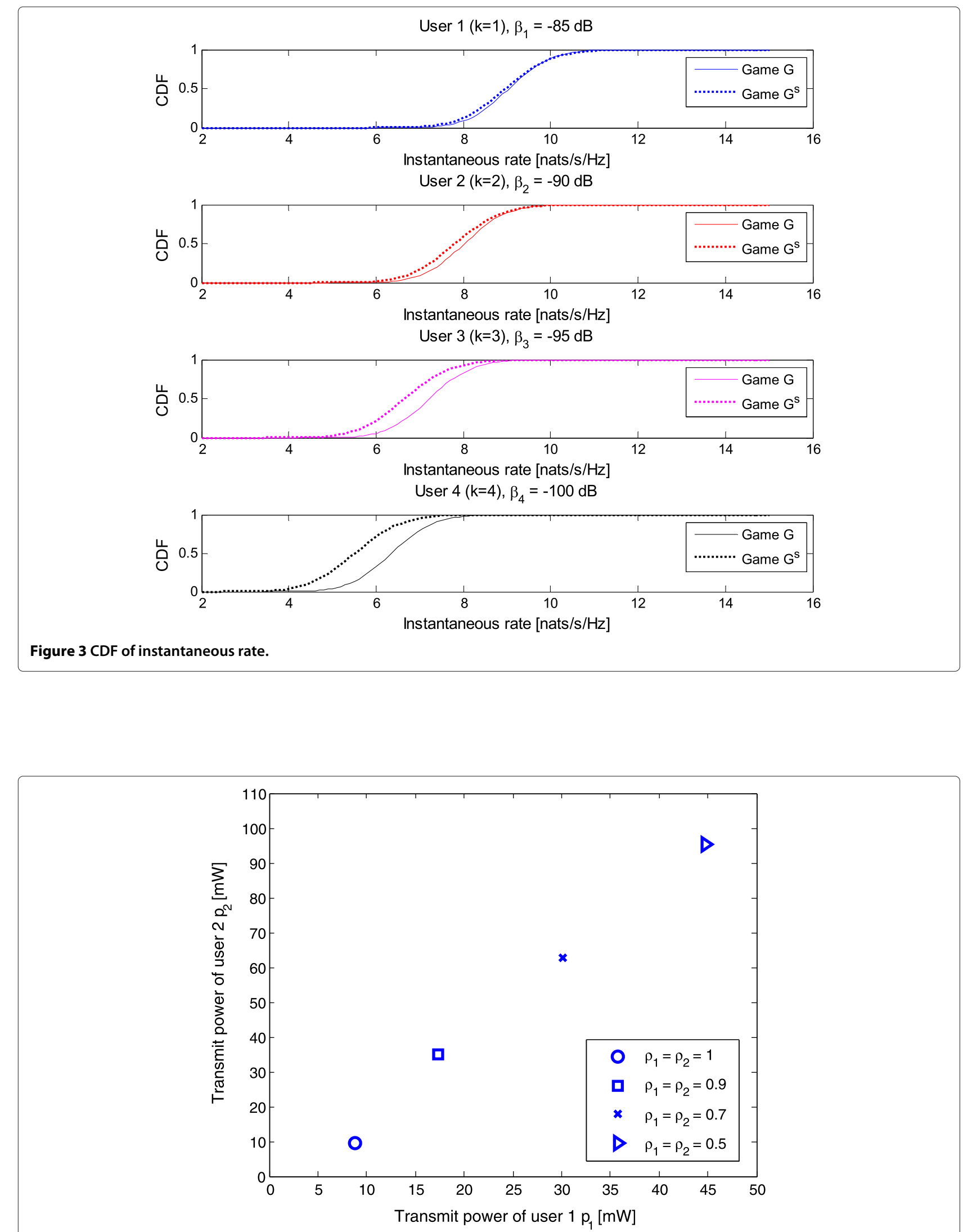

Figure 4 Nash equilibrium of the game $\mathcal{G}$ for different channel certainties $\rho_{\boldsymbol{k}} \mathbf{s} . M=8, K=2$, and $\beta_{1}=-90 \mathrm{~dB}, \beta_{2}=-95 \mathrm{~dB}$. 


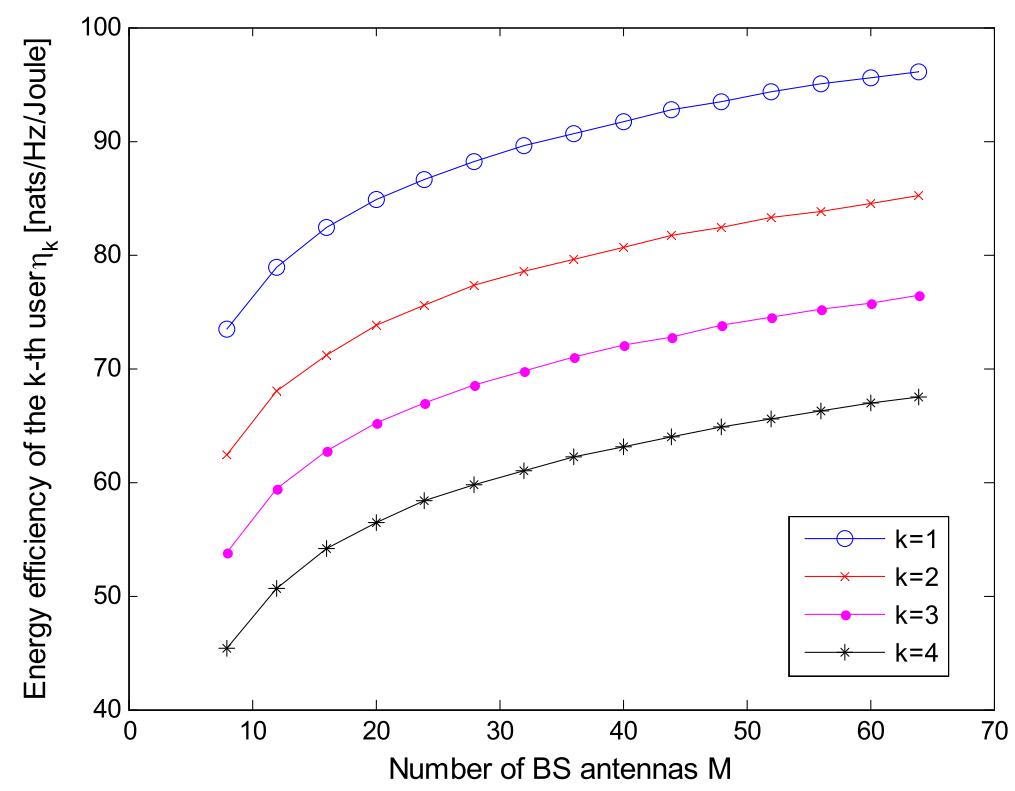

Figure $5 \mathrm{EE}$ at the Nash equilibrium versus the number of $\mathrm{BS}$ antennas. $K=4$ and $\beta_{1}=-85 \mathrm{~dB}, \beta_{2}=-90 \mathrm{~dB}, \beta_{3}=-95 \mathrm{~dB}, \beta_{4}=-100 \mathrm{~dB}$ and $\rho_{1}=\rho_{2}=\rho_{3}=\rho_{4}=0.9995$.

follows. Since $\log x$ is an increasing function in $x>0$, the best response of the proposed scheme in (11) can be equivalently written as

$$
\begin{aligned}
p_{d}^{o} & =\arg \max _{0 \leq p_{k} \leq p_{\max }} \log \left(\eta_{k}\left(p_{k}, \mathbf{p}_{-k}\right)\right) \\
& =\arg \max _{0 \leq p_{k} \leq p_{\max }} \log R_{k}^{\text {lower }}-\log \left(p_{k}+p_{c}\right),
\end{aligned}
$$

which implies that the energy-efficient uplink power control game $\mathcal{G}$ can be regarded as a variation of the spectralefficient game $\mathcal{G}^{s}$ with a pricing. Since the game $\mathcal{G}$ applies a penalty to a power consumption, the players in $\mathcal{G}$ tend to use the power in a conservative way. This reduces the interference to the other players and the average rate of the users can be improved.

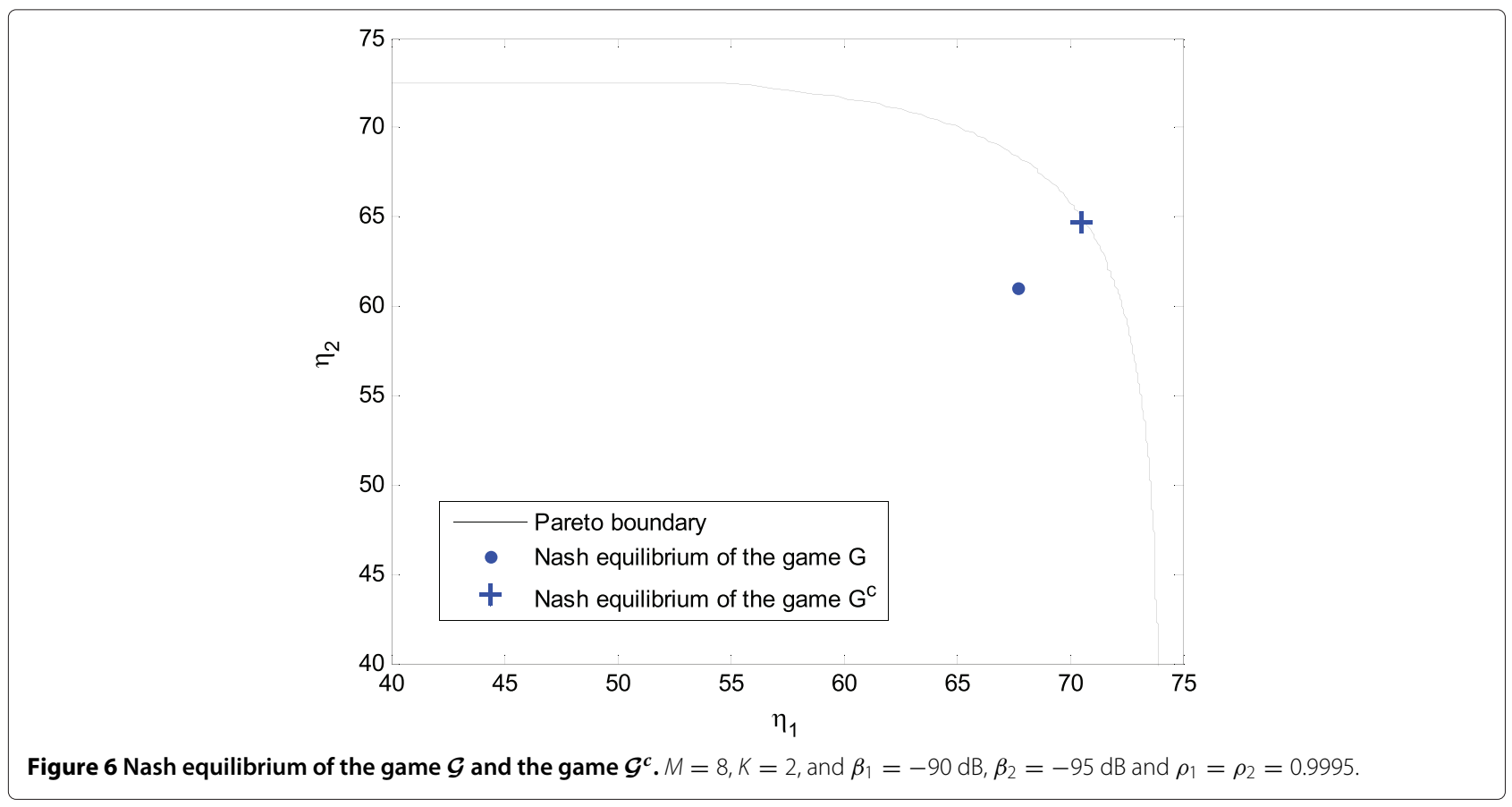




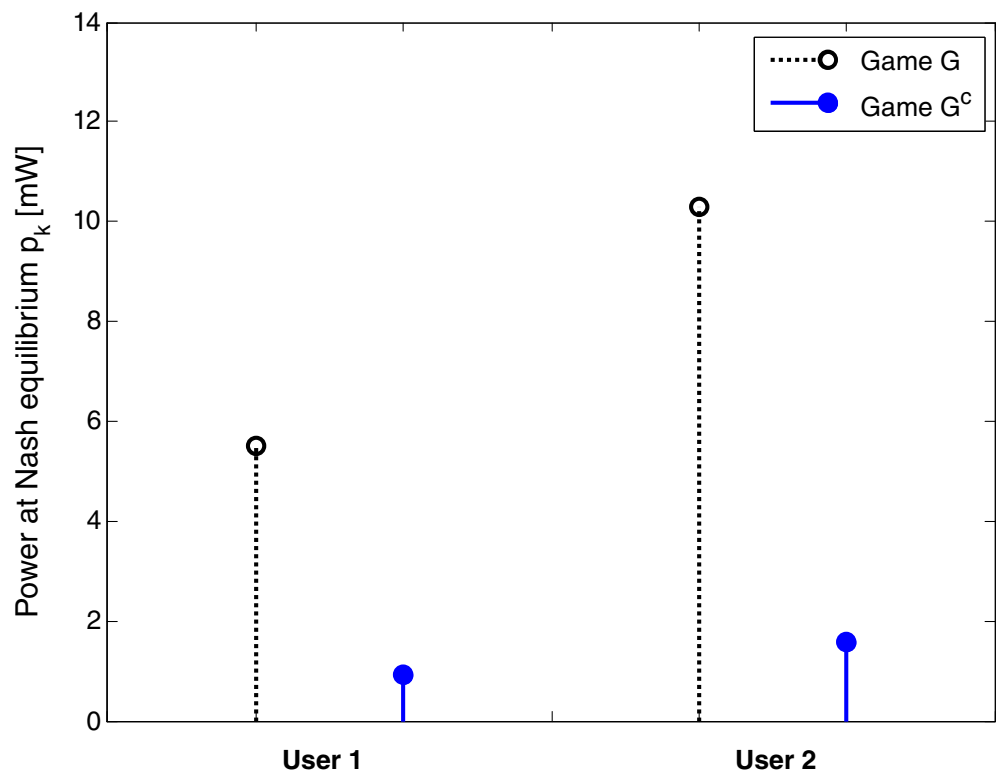

Figure 7 Transmit powers of users at the Nash equilibria.

Figure 4 considers $K=2$ users and plots their transmit powers and EEs obtained by the best response dynamics of the proposed game for different values of channel certainty measure in correlation coefficient $\rho_{k}$. As shown in the figure, when the BS has higher $\rho_{k} \mathrm{~s}$, less transmit powers are required and higher EEs can be achieved for the users. Figure 5 shows the EE versus the number of BS antennas. Since the receive beamforming gain increases as the number of transmit antennas increases, the EEs of the users increase accordingly.

Figures 6 and 7 show the effect of the pricing on the Nash equilibrium of the game $\mathcal{G}^{c}$. Figure 6 plots the Pareto boundary of $\left(\eta_{1}, \eta_{2}\right)$ and locates the Nash equilibriums of the game $\mathcal{G}$ and the game $\mathcal{G}^{c}$. Figure 7 shows the corresponding transmit powers of the users. The Nash equilibrium of the game $\mathcal{G}^{c}$ achieves a Pareto improvement over that of the game $\mathcal{G}$. This increase in the efficiency of the Nash equilibrium is due to the fact that the players of the game $\mathcal{G}^{c}$ spend less power motivated by the price than those in the game $\mathcal{G}$, which is more favorable to the other players' EE.

\section{Conclusions}

We have considered energy-efficient transmit power control for uplink multiuser SIMO systems when the BS has imperfect CSI using a game-theoretic approach. The proposed energy-efficient uplink power control game is shown to have at least one Nash equilibrium. Furthermore, the uniqueness of the Nash equilibrium as well as the convergence of the best response dynamics is shown. To improve the efficiency of the Nash equilibrium, we propose a new game that utilizes a pricing mechanism. For the new game, the existence and the convergence of the best response dynamics is also investigated by using the super-modularity theory. Finally, we propose a simple algorithm to find the pricing factor that improves the system performance in the Pareto sense. From the simulation results, we can see that the proposed energy-efficient power control schemes significantly enhance the EE of the users in uplink multiuser SIMO systems.

\section{Endnotes}

${ }^{a}$ In this paper, metrics are computed in units of nats/s to simplify the expressions and analysis, with 1 nats $/ \mathrm{s}=$ $1.443 \mathrm{bits} / \mathrm{s}$.

${ }^{\mathrm{b}}$ There are some low-complexity optimization methods that can reduce the complexity of the exhaustive search. For example, monotonic optimization [33] effectively reduces the feasible region when the object function to be maximized is increasing.

\section{Appendices}

\section{Appendix 1}

\section{Proof of Theorem 1}

Using Jensen's inequality [34] and the convexity of $\log \left(1+\frac{1}{x}\right)$, we have a lower bound of $R_{k}$,

$$
R_{k}=E\left[\log \left(1+\frac{1}{\frac{1}{\gamma_{k}}}\right)\right] \geq \log \left(1+\frac{1}{E\left[\frac{1}{\gamma_{k}}\right]}\right) .
$$


From (2) and the independence of $\hat{\mathbf{h}}_{k}$ and $\mathbf{e}_{j}$, we have

$$
\begin{aligned}
\frac{1}{E\left[\frac{1}{\gamma_{k}}\right]}= & \underbrace{E\left[c_{k}\left|\mathbf{a}_{k}^{T} \mathbf{e}_{k}\right|^{2} p_{k}+\sum_{j \neq k} c_{j}\left|\mathbf{a}_{k}^{T} \mathbf{e}_{j}\right|^{2} p_{j}+1\right]}_{(a)} \\
& \times \underbrace{E\left[\frac{1}{c_{k}\left|\rho_{k}\right|^{2}\left|\mathbf{a}_{k}^{T} \hat{\mathbf{h}}_{k}\right|^{2} p_{k}}\right]}_{(b)} .
\end{aligned}
$$

Using (3), (a) can be written as

$$
(a)=c_{k}\left(1-\rho_{k}^{2}\right) p_{k}+\sum_{j \neq k} c_{j}\left(1-\rho_{j}^{2}\right) p_{j}+1 .
$$

From (4), we can see that $(b)$ is the expectation of an inverse gamma distributed random variable [35]. Therefore, $(b)$ can be written as

$$
(b)=\frac{1}{(M-K) c_{k} \rho_{k}^{2} p_{k}} .
$$

From (17) to (19), (16) is given by

$$
R_{k}^{\text {lower }}=\log \left(1+\frac{s_{k} p_{k}}{i_{k} p_{k}+\sum_{j \neq k} i_{j} p_{j}+1}\right)
$$

where $s_{k}=(M-K) c_{k} \rho_{k}^{2}$ and $i_{k}=c_{k}\left(1-\rho_{k}^{2}\right)$.

\section{Appendix 2}

\section{Proof of Theorem 2}

We find the modified strategy space $\mathcal{Q}_{k}^{c}$ that makes the game $\mathcal{G}^{c}$ satisfy condition 3), i.e., we find $\mathcal{Q}_{k}^{c}$ such that

$$
g\left(p_{k}\right)=\eta_{k}^{c}\left(p_{k}, \mathbf{p}_{-k}\right)-\eta_{k}^{c}\left(p_{k}, \mathbf{p}_{-k}^{\prime}\right)
$$

is non-decreasing in $p_{k} \in \mathcal{Q}_{k}^{c}$ for $\mathbf{p}_{-k} \geq \mathbf{p}_{-k}^{\prime}$. Note that (20) can be written as

$$
g\left(p_{k}\right)=\frac{w\left(p_{k}\right)}{v\left(p_{k}\right)}
$$

where $v\left(p_{k}\right)=p_{k}+p_{c}$ and

$$
w\left(p_{k}\right)=\log \left(1+\frac{s_{k} p_{k}}{i_{k} p_{k}+I_{k}}\right)-\log \left(1+\frac{s_{k} p_{k}}{i_{k} p_{k}+I_{k}^{\prime}}\right)
$$

with $I_{k}=\sum_{j=1, j \neq k}^{K} i_{j} p_{j}+1$ and $I_{k}^{\prime}=\sum_{j=1, j \neq k}^{K} i_{j} p_{j}^{\prime}+1$. We observe that $\frac{1}{v\left(p_{k}\right)}$ is positive and decreasing in $p_{k}>0$.
Also, we observe that $w\left(p_{k}\right)<0$ for $p_{k}>0$ because $I_{k} \geq I_{k}^{\prime}$ for $\mathbf{p}_{-k} \succeq \mathbf{p}_{-k}^{\prime}$. Then, we find $\mathcal{Q}_{k}^{c}$ such that $w\left(p_{k}\right)$ is increasing in $p_{k} \in \mathcal{Q}_{k}^{c}$, i.e., $\frac{\partial w}{\partial p_{k}} \geq 0$, or equivalently,

$$
a p_{k}^{2}+2 b p_{k}+c \geq 0
$$

for $p_{k} \in \mathcal{Q}_{k}^{c}$, where $a=s_{k} i_{k}\left(I_{k}-I_{k}^{\prime}\right), b=i_{k}\left(I_{k}^{\prime}-I_{k}\right)$, and $c=I_{k}^{\prime 2}-I_{k}^{2}$. By solving the quadratic Equation in (22), we see that if

$$
p_{k} \geq \delta_{k} \triangleq \frac{i_{k}+\sqrt{i_{k}^{2}+s_{k} i_{k}\left(I_{k}+I_{k}^{\prime}\right)}}{s_{k} i_{k}}
$$

then $b\left(p_{k}\right)$ is increasing. Since $I_{k}+I_{k}^{\prime} \leq 2 I_{\max , k}$, where $I_{\max , k}=\sum_{j=1, j \neq k}^{K} i_{j} p_{\max }+1$, we have

$$
p_{\min , k} \triangleq \frac{i_{k}+\sqrt{i_{k}^{2}+2 s_{k} i_{k} I_{\max , k}}}{s_{k} i_{k}} \geq \delta_{k} .
$$

Since 1) $\frac{1}{v\left(p_{k}\right)}>0$ is decreasing in $p_{k}>0$ and 2) $w\left(p_{k}\right)<$ 0 is increasing in $p_{k} \geq p_{\min , k}$, it is clear that $g\left(p_{k}\right)$ is nondecreasing in $p_{k} \geq p_{\min , k}$.

Competing interests

The authors declare that they have no competing interests.

\section{Acknowledgements}

This research was supported by the Basic Science Research Program through the National Research Foundation of Korea (NRF) funded by the Ministry of Education (2013R1A1A2012202).

Received: 30 December 2013 Accepted: 1 October 2014

Published: 12 October 2014

\section{References}

1. AJ Paulraj, DA Gore, RU Nabar, H Bolcskel, An overview of MIMO communications - a key to gigabit wireless. Proc. IEEE. 92(2), 198-218 (2002)

2. G Caire, S Shamai, On the achievable throughput of a multiantenna Gaussian broadcast channel. IEEE Trans. Inf. Theroy. 49(7), 1691-1706 (2003)

3. H Weingarten, Y Steinberg, S Shamai, The capacity region of the Gaussian multiple-input multiple-output broadcast channel. IEEE Trans. Inf. Theroy. 52(9), 3936-3964 (2006)

4. CB Peel, BM Hochwald, AL Swindlehurst, A vector-perturbation technique for near capacity multiantenna multiuser communication-part l: channel inversion and regularization. IEEE Trans. Commun. 53(1), 195-202 (2005)

5. BM Hochwald, CB Peel, AL Swindlehurst, A vector-perturbation technique for near capacity multiantenna multiuser communication-part II: perturbation. IEEE Trans. Commun. 53(3), 537-544 (2005)

6. TYoo, A Goldsmith, On the optimality of multiantenna broadcast scheduling using zero-forcing beamforming. IEEE J. Sel. Areas Commun. 24(3), 528-541 (2006) 
7. Z Hasan, H Boostanimehr, VK Bhargava, Green cellular networks: a survey, some research issues and challenges. IEEE Commun. Surv. Tutorials. 13(4), 524-540 (2011)

8. RS Prabhu, B Daneshrad, Energy-efficient power loading for a MIMO-SVD system and its performance in flat fading, in Proc. Global Telecommun. Conf (Miami, 6 Dec 2010), pp. 1-5

9. EV Belmega, S Lasaulce, Energy-efficient precoding for multiple-antenna terminals. IEEE Trans. Signal. Process. 59(1), 329-340 (2011)

10. C Jiang, L Climini, Antenna selection for energy-efficient MIMO transmission. IEEE Wireless Commun. Lett. 1(6), 577-580 (2012)

11. H Kim, CB Chae, G Veciana, RW Heath Jr., A cross-layer approach to energy efficiency for adaptive MIMO systems exploiting spare capacity. IEEE Trans. Wireless Commun. 8(8), 4264-4275 (2009)

12. C Hellings, N Damak, W Utschick, Energy-efficient zero-forcing with user selection in parallel vector broadcast channels, in International ITG Workshop on Smart Antennas (Dresden, 7 Mar 2012), pp. 168-175

13. G Miao, Energy-efficient uplink multi-user MIMO. IEEE Trans. Wireless Commun. 12(5), 2302-2313 (2013)

14. J Xu, L Qiu, Energy efficiency optimization for MIMO broadcast channels. IEEE Trans. Wireless Commun. 12(2), 690-701 (2013)

15. Y Rui, QT Zhang, L Deng, P Cheng, M Li, Mode selection and power optimization for energy efficiency in uplink virtual MIMO systems. IEEE J. Sel. Areas Commun. 31(5), 926-936 (2013)

16. S Buzzi, HV Poor, D Saturino, Energy-efficient resource allocation in multiuser MIMO systems: a game-theoretic framework, in Proc. of the 16th European Signal Processing Conference (EUSIPCO 2008) (Lausanne, 25 Aug 2008)

17. Y Nam, L Liu, G Miao, CJ Zhang, Link adaptation for energy-efficient uplink coordinated multi-point receptions. EURASIP J. Wireless Commun. Networking. 2011(1), 1-8 (2011)

18. Z Chong, E Jorswieck, Energy-efficient power control for MIMO time-varying channels, in Online Conference on Green Communications (GreenCom), (26 Sept 2011), pp. 92-97

19. GW Miao, N Himayat, GY Li, S Talwar, Distributed interference-aware energy-efficient power optimization. IEEE Trans. Wireless Commun. 10(4), 1323-1333 (2011)

20. A Zappone, G Alfano, S Buzzi, M Meo, Distributed energy-aware resource allocation in multi-antenna multi-carrier interference networks with statistical CSI. EURASIP J. Wireless Commun. Networking. 2013(1), 1-16 (2013)

21. J Xu, L Qiu, C Yu, Improving energy efficiency through multimode transmission in the downlink MIMO systems. EURASIP J. Wireless Commun. Networking. 2011(1), 1-12 (2011)

22. Y Isukapalli, R Annavajjala, BD Rao, Performance analysis of transmit beamforming for MISO systems with imperfect feedback. IEEE Trans. Commun. 57(1), 222-231 (2009)

23. H Poor, An Introduction to Signal Detection and Estimation. (Springer-Verlag, New York, 1994)

24. J Zhang, JG Andrews, Adaptive spatial intercell interference cancellation in multicell wireless networks. IEEE J. Sel. Areas Commun. 28(9), 1455-1468 (2010)

25. F Rusek, D Persson, BK Lau, EG Larsson, TL Marzetta, O Edfors, F Tufvesson, Scaling up MIMO: opportunities and challenges with very large arrays. IEEE Signal Process. Mag. 30(1), 40-46 (2013)

26. HQ Ngo, EG Larsson, TL Marzetta, Energy and spectral efficiency of very large multiuser MIMO systems. IEEE Trans. Commun. 61(3), 1436-1449 (2013)

27. S Cui, AJ Goldsmith, A Bahai, Energy-constrained modulation optimization. IEEE Trans. Wireless Commun. 4(5), 2349-2360 (2005)

28. JF Nash, Non-cooperative games. Ann. Math. 54, 289-295 (1951)

29. A Zappone, Z Chong, E Jorswieck, S Buzzi, Energy-aware competitive power control in relay-assisted interference wireless networks. IEEE Trans. Wireless Commun. 12(4), 1860-1871 (2013)

30. CU Saraydar, NB Mandayam, DJ Goodman, Efficient power control via pricing in wireless data networks. IEEE Trans. Commun. 50(2), 291-303 (2002)

31. DM Topkis, Equilibrium points in nonzero sum n-person submodular games. SIAM J. Control Optim. 17(6), 773-787 (1979)

32. DM Topkis, Supermodularity and Complementarity. (Princeton Univ. Press, Princeton, 1998)
33. YJ Zhang, L Qian, J Huang, Monotonic optimization in communication and networking systems. Found Trends $®$ Networking. 7(1), 1-75 (2013)

34. TM Cover, JA Thomas, Elements of Information Theory. (Wiley, New York, 1991)

35. AD Dabbagh, DJ Love, Multiple antenna MMSE based downlink precoding with quantized feedback or channel mismatch. IEEE Trans. Commun. 56(11), 1859-1868 (2008)

doi:10.1186/1687-1499-2014-166

Cite this article as: Jang et al.: Energy-efficient uplink power control for multiuser SIMO systems with imperfect channel state information. EURASIP Journal on Wireless Communications and Networking 2014 2014:166.

\section{Submit your manuscript to a SpringerOpen ${ }^{\circ}$ journal and benefit from:}

- Convenient online submission

- Rigorous peer review

- Immediate publication on acceptance

- Open access: articles freely available online

- High visibility within the field

- Retaining the copyright to your article

Submit your next manuscript at $\boldsymbol{\nabla}$ springeropen.com 